\section{The impact of a misinterpretation of the term "overtreatment"}

\section{Dear Editor,}

Collective screening identifying endocrine diseases has not only the advantage of achieving early diagnosis but also the disadvantage of leading to overdiagnosis. Overdiagnosis involves the identification of diseases that would not have been diagnosed throughout the life course without screening. Therefore, diagnosis and treatment would actually be unnecessary for overdiagnosed patients throughout their life, and would also confer major disadvantages for them, not only physically but also psychosocially. When overdiagnosis of cancer occurs, the pathological diagnosis itself is correct, so overdiagnosis is completely different from misdiagnosis. Treatment in cases of overdiagnosis can be defined as overtreatment [1].

Clinicians may underestimate the disadvantages of overdiagnosis and overtreatment because it is not known in individual cases whether the diagnosis involves overdiagnosis or early detection that would benefit the patient [2]. There is also confusion in the use of the terms "overdiagnosis" and "overtreatment," which obscures the harm inflicted when these occur. As a representative case of this, we would like to point out a couple of seriously misleading descriptions in the discussion of the article by Sakamoto et al. [3].

First, there is a misinterpretation of the term "overtreatment". Sakamoto et al. [3] concluded that "problems concerning overtreatment basically do not occur in this survey" based on the cited paper, not on their analyzed data. They used the term "overtreatment" here simply to mean not performing excessive surgical treatment. However, the NCI Dictionary of Cancer Terms defines "overtreatment" as follows: "Treatment of a cancer that would have gone away on its own or never caused any symptoms. These cancers are usually found on a screening test. Overtreatment may lead to problems and harmful

Sanae Midorikawa ${ }^{1)}$ and Akira Ohtsuru ${ }^{2)}$

1) Miyagi Gakuin Women's University, Sendai 981-8557, Japan

2) Atomic Bomb Disease Institute, Nagasaki University, Nagasaki 852-8523, Japan

Submitted Sep. 20, 2020; Accepted Oct. 20, 2020 as EJ20-0604

Released online in J-STAGE as advance publication Nov. 21, 2020

Correspondence to: Sanae Midorikawa, Miyagi Gakuin Women's University, 9-1-1 Sakuragaoka, Aobaku, Sendai 981-8557, Japan.

E-mail: s-midori@mgu.ac.jp side effects of cancer therapies that are not needed" [1]. Thus, overtreatment generally means to treat diseases that are overdiagnosed, but it does not mean to excessively perform medical or surgical techniques. It is difficult to evaluate the exact rate of overdiagnosis, but some previous reports pointed out that there would be a significant number of potential overdiagnoses in this screening program. Katanoda et al. reported that the cumulative prevalence in the first round was 31 times higher than that without screening [4]. Assuming that all of these cancers might be diagnosed early as future clinical cancers rather than be overdiagnosed, the latent period before clinical diagnosis was estimated to be 34 and 30 years in men and women, respectively [5]. Furthermore, in the second round 2 years later, the incidence rate of thyroid cancer was reported to be more 30 times higher than that without screening [6]. A total of 186 children or young adults were diagnosed with thyroid cancer in both rounds including around 300,000 subjects. With the progress of diagnostic imaging since around 1980, the rate of thyroid cancer overdiagnosis has been gradually increasing in the world without population screening [7]. As references, the cumulative incidence of thyroid cancer in 1975 (when it is considered that there was no overdiagnosis in Japan) was around 37 per 100,000 from age 0 to 49 years [8]. Even if all cases of thyroid cancer by age 49 were diagnosed by these two tests, even more thyroid cancers are suspected to be diagnosed in these screenings. Considering these reports comprehensively, it must be said that a significant number of thyroid cancers have been overdiagnosed.

Active surveillance in young people under the confusing situation after the nuclear accident is difficult to select. We have simulated the growth pattern of thyroid cancer from the data before surgery and found that most of these cases would fall into growth arrest after the initial proliferation [9]. Therefore, there is no evidence that the presence or absence of a pathological extrathyroidal extension and/or lymph node metastasis is an indicator of prognosis. Overtreatment would inevitably have occurred. The misuse of medical terminology in the article by Sakamoto et al. [3] can lead to the misunderstanding among readers that there are very few cases of overdiagnosis and overtreatment, and should be corrected. This survey may be able to diagnose a few patients that will develop clinical cancers in a few years. However, the benefits of early diagnosis in thyroid cancer have not been proven [10] and some harm has been reported, such as a potential increase in the number of surgeries [11]. 
We also reported that extremely early diagnosis may cause physical, psychological, economical, and social disadvantages, like overdiagnosis [12]. Furthermore, there seems to be no evidence that postoperative pathological findings of thyroid cancer, such as extrathyroidal invasion or lymph node metastasis, can determine whether clinical cancer will develop within a few years. In the first place, it is impossible to judge whether the patients were overtreated from the postoperative pathological findings. Moreover, cytological findings in this paper by Sakamoto et al. [3] cannot discriminate clinical cancer and indolent cancer.

As a second issue, there is an incomplete citation regarding the recommendation for screening of thyroid cancer after radiation exposure. Although the US Preventive Services Task Force recommendation was stated not to apply to those at increased risk of thyroid cancer due to a history of exposure to ionizing radiation by medical treatment or radiation fallout [10], the threshold of the dose of fallout exposure at which this recommendation does not apply is not stated. The statistical risk of thyroid cancer upon radiation exposure depends on the radiation dose, which gradually increases from around $100 \mathrm{mSv}$ or higher. Because the radiation dose among Fukushima residents is extremely low, an association between the large number of thyroid cancers detected in this survey and radiation exposure is thought to be very unlikely [13]. Furthermore, the WHO/IARC report recommends against population thyroid screening after a nuclear accident [14]. Owing to the harm resulting from overdiagnosis, a recent review article also does not support the implementation of an ultrasound-based screening program even in high-risk populations (those with hereditary syndromes, high-dose radiation therapy, or children exposed to nuclear fallout, etc.) $[14,15]$. The discussion in this article would lead to the misunderstanding that the radiation dose among Fukushima residents may be high enough to cause radiation-induced thyroid cancer or that population screening is more beneficial than harmful in an area with radiation fallout at any dose.

In the early years after the nuclear accident, this screening program was launched to address the anxieties of Fukushima residents. However, unfortunately the results of this screening have increased anxiety among residents and their risk perception of radiation exposure. To address anxieties about health risks of radiation exposure, we need to respond to those who are really anxious and to monitor them individually, instead of conducting large-scale thyroid cancer screening using ultrasound. Specifically, we recommend the minimum required is as follows: 1) to target subjects who have applied with an opt-in method (not for all residents, not school examinations); 2) to communicate with participants regarding factors associated with their anxieties, radiation exposure dose, and harms of thyroid ultrasound examination before performing the examination; and 3) to suppress cytological examination to avoid overdiagnosis.

The authors who analyzed the results of the Fukushima Health Management Survey should be careful to understand the above background and to avoid misleading readers about overdiagnosis or overtreatment.

\section{Acknowledgments}

We thank Edanz (https://en-author-services.edanzgroup. com/ac) for editing the English text of a draft of this manuscript.

\section{References}

1. NCI Dictionary of Cancer Terms: Overtreatment. National Cancer Institute. https://www.cancer.gov/publications/ dictionaries/cancer-terms/def/overtreatment accessed on September 20, 2020.

2. Raffle A, Gray M (2007) Screening: evidence and practice. Oxford University Press, Oxford, UK.

3. Sakamoto A, Matsuzuka T, Yamaya Y, Suzuki S, Iwadate $\mathrm{M}$, et al. (2020) Cytological examination of the thyroid in children and adolescents after the Fukushima Nuclear Power Plant accident: the Fukushima Health Management Survey. Endocr J Epub ahead of print.

4. Katanoda K, Kamo KI, Tsugane S (2016) Quantification of the increase in thyroid cancer prevalence in Fukushima after the nuclear disaster in 2011 - a potential overdiagnosis? Jpn J Clin Oncol 46: 284-286.

5. Takahashi H, Takahashi K, Shimura H, Yasumura S, Suzuki S, et al. (2017) Simulation of expected childhood and adolescent thyroid cancer cases in Japan using a cancer-progression model based on the National Cancer Registry: application to the first-round thyroid examination of the Fukushima Health Management Survey. Medicine 96: e8631.

6. Ohtsuru A, Midorikawa S, Ohira T, Suzuki S, Takahashi $\mathrm{H}$, et al. (2019) Incidence of thyroid cancer among children and young adults in Fukushima, Japan, screened with 2 rounds of ultrasonography within 5 years of the 2011 Fukushima Daiichi Nuclear Power Station accident. JAMA Otolaryngol Head Neck Surg 145: 4-11.

7. Welch HG, Kramer BS, Black WC (2019) Epidemiologic signatures in cancer. New Engl J Med 381: 1378-1386.

8. Cancer Registry and Statistics. Cancer Information Service, National Cancer Center, Japan (Ministry of Health, Labour and Welfare, National Cancer Registry) https:// ganjoho.jp/reg_stat/statistics/dl/index.html (October 22, 2020)

9. Midorikawa S, Ohtsuru A, Murakami M (2018) Comparative analysis of the growth pattern of thyroid cancer in young patients screened by ultrasonography in Japan after a nuclear accident: The Fukushima Health Management 
Survey. JAMA Otolaryngol Head Neck Surg 144: 57-63.

10. US Preventive Services Task Force (2017) Screening for thyroid cancer: US Preventive Services Task Force recommendation statement. JAMA 317: 1882-1887.

11. Lin JS, Aiello Bowles EJ, Williams SB, Morrison CC (2017) Screening for thyroid cancer: Updated evidence report and systematic review for the US Preventive Services Task Force. JAMA 317: 1888-1903.

12. Midorikawa S, Murakami M, Ohtsuru A (2019) Harm of overdiagnosis or extremely early diagnosis behind trends in pediatric thyroid cancer. Cancer 125: 4108-4109.

13. UN Scientific Committee on the Effects of Atomic Radiation. Sources. Developments since the 2013 UNSCEAR report on the levels and effects of radiation exposure due to the nuclear accident following the Great East Japan earthquake and tsunami: a 2017 white paper to guide the scientific committee's future programme of work. New York: United Nations, 2017. https://www.unscear.org/ docs/publications/2017/UNSCEAR_WP_2017.pdf accessed on September 20, 2020.

14. Togawa K, Ahn HS, Auvinen A, Bauer AJ, Brito JP, et al. (2018) Long-term strategies for thyroid health monitoring after nuclear accidents: recommendations from an Expert Group convened by IARC. Lancet Oncol 19: 1280-1283.

15. Lamartina L, Grani G, Durante C, Filetti S, Cooper DS (2020) Screening for differentiated thyroid cancer in selected populations. Lancet Diabetes Endocrinol 8: 81-88. 